\title{
Article \\ Performance Analysis of Wireless Local Area Network for a High-/Low-Priority Traffic Ratio at Different Numbers of Access Categories
}

\author{
Kvitoslava Obelovska ${ }^{1, *}$, Olga Panova ${ }^{1}$ and Vincent Karovič, Jr. ${ }^{2}$ \\ 1 Department of Automated Control Systems, Lviv Polytechnic National University, 79013 Lviv, Ukraine; \\ olga.panova@gmx.at \\ 2 Department of Information Systems, Faculty of Management, Comenius University, Odbojárov 10, \\ 81499 Bratislava, Slovakia; vincent.karovicml@fm.uniba.sk \\ * Correspondence: kvitoslava.m.obelovska@lpnu.ua
}

check for updates

Citation: Obelovska, K.; Panova, O.; Karovič, V., Jr. Performance Analysis of Wireless Local Area Network for a High-/Low-Priority Traffic Ratio at Different Numbers of Access Categories. Symmetry 2021, 13, 693. https://doi.org/10.3390/sym13040693

Academic Editors: Ivan Izonin,

Stephane Chretien and

Ali Ebrahimnejad

Received: 4 March 2021

Accepted: 6 April 2021

Published: 15 April 2021

Publisher's Note: MDPI stays neutral with regard to jurisdictional claims in published maps and institutional affiliations.

Copyright: (c) 2021 by the authors. Licensee MDPI, Basel, Switzerland. This article is an open access article distributed under the terms and conditions of the Creative Commons Attribution (CC BY) license (https:/ / creativecommons.org/licenses/by/ $4.0 /)$.

\begin{abstract}
The performance of Wireless Local Area Network (WLAN) is highly dependent on the processes that are implemented in the Medium Access Control (MAC) sublayer regulated by the IEEE 802.11 standard. In turn, various parameters affect the performance of the MAC sublayer, the most important of which is the number of stations in the network and the offered load. With the massive growth of multimedia traffic, research of the network performance depending on traffic types is relevant. In this paper, we present the impact of a high-/low-priority traffic ratio on WLAN performance with different numbers of access categories. The simulation results show different impact of high-/low-priority traffic ratio on the performance of the MAC sublayer of wireless LANs depending on different network-sizes and on network conditions. Performance of the large network with two access categories and with the prevalent high-priority traffic is significantly higher than in the case of using four categories on the MAC sublayer. This allows us to conclude that the performance improvement of the large network with the prevalent high-priority traffic can be achieved by an adaptive adjustment of the access categories number on the MAC sublayer.
\end{abstract}

Keywords: wireless local area networks; MAC sublayer; EDCA

\section{Introduction}

Searching for new ways to improve Wireless Local Area Network (WLAN) performance is caused by a burst increase of Wi-Fi-enabled devices and intense growing demand for high-quality transmission of multimedia content. WLAN uses a shared physical environment to exchange data between active devices. Each node in a network can claim to share a channel; therefore, one of the important problems of WLANs is controlling access to the physical environment. In the most common wireless Wi-Fi networks [1], station access to the environment is competitive using the Carrier Sense Multiple Access with Collision Avoidance (CSMA/CA). Using of a part of the network bandwidth to implement the CSMA/CA reduces a network bandwidth for transmission of subscriber traffic. Thus, this negatively affects wireless network performance, and improvement of accessing the physical environment methods of wireless computer networks plays an important role in the functioning of the Medium Access Control (MAC) sublayer.

Access to the physical environment of WLANs is regulated by the IEEE 802.11 standard [1]. The most widely used MAC protocol is based on the CSMA/CA scheme. This scheme provides contention-based access to the physical environment. In the early versions of IEEE 802.11 the CSMA/CA mechanism was implemented as the Distributed Coordination Function (DCF). DCF provides equal access for all nodes in the network by using a time-dependent set of parameters: slot time, inter-frame space, and Contention Window ranged from its minimum to its maximum value. Besides, in Access Point-oriented networks, the IEEE 802.11 standard provides Point Coordination Function (PCF) that enables 
centralized access to the physical environment. However, unlike DCF, which is necessary and important, $\mathrm{PCF}$ is optional and not all manufacturers support it.

Distributed Coordination Function does not support Quality of Services (QoS). To provide multimedia applications and real-time traffic an Enhanced Distributed Channel Access (EDCA) is developed. Unlike the basic DCF scheme, the EDCA scheme, which is an extension of it, designed to support Quality of Service and supports four categories of access. The EDCA scheme defines four access categories (ACs) that provide support for the delivery of prioritized traffic. Each $\mathrm{AC}$ has a transmission queue and independently executes DCF scheme. As the higher-priority frames now have a better chance to be transmitted, the channel utilization is reduced. Many research works [2-10] are aimed to improve the performance of MAC sublayer by using different methods and research approaches $[2,4,5]$. Moreover, most of them aims to ensure the transmission of various traffic types with different requirements, however, the impact of different ratios of various traffic types on the network performance has not yet been studied. Knowledge of this impact would provide an opportunity to develop specialized algorithms to improve the MAC sublayer oriented on certain ratios of traffic types in each specific application. As a result, network efficiency could be enhanced.

The main contributions of this paper can be summarized as follows:

- It is revealed that the impact of high-/low-priority traffic ratio on the MAC sublayer performance of wireless LANs depends on the number of access categories and is different for different network sizes;

- It is also shown that, for a large network, the impact of high-/low-priority traffic ratio is significant. In the case of prevailed high-priority network load the total network capacity for intensive load is almost twice less, than in the case of prevailed low-priority network load;

- Based on the simulation, the conditions, under which the adaptive adjusting of the number of access categories could significantly increase the efficiency of wireless LAN, are determined.

\section{Related Works}

Nowadays, many works are devoted to study and improve the access method to the physical environment defined by IEEE 802.11 standard. A variety of research methods are used, for example, network simulator [2]; Markov chain [3]; Colored Petri Net [4]; machine learning [5]; to provide QoS to specific flows in network traffic, such as voice [11]; video [12]; downloading large files [13]; signaling [14], and integrated traffic [15].

Guaranteeing the quality of service as providing sufficient level of service to meet the traffic requirements is one of the key research areas to improve wireless network performance. A general guide to improving QoS for communication system engineers is presented in Reference [16]. The actual and future trends of QoS wireless networks are discussed in Reference [17]. There are also QoS improvement approaches for specific network types-End-to-End QoS management algorithms for Internet of Things Services in 4G/5G Networks [18], or instilling QoS in Wireless Sensor Networks [7].

Enhancing the quality of service can be also achieved by improving the MAC sublayer. For example, the proposed modified MAC protocol for nano-networks improves their performance and energy efficiency, reduces the probability of collisions [6]. Reference [8] presents a comprehensive review on the techniques to improve the BEB algorithm used to retransmission of frames in CSMA/CA scheme of wireless local area networks. In Reference [9], incoming traffic is divided into three categories, namely normal, urgent, and critical, to which different priorities and different delay times for each category are assigned. It improves the transmission of urgent and critical data. IEEE 802.11 networks enable the QoS by combining the DCF and EDCA with static parameters regardless of existing network capacity, the number of associated stations, or the activity of Access Categories. Moreover, as it has been shown in References [19,20], in case of a highly loaded network, this leads to significant network performance degradation. 
As the throughput of WLANs is bounded by the overhead introduced in the MAC protocol $[15,21]$ some of the studies are focused on optimizing and maximizing channel capacity and utilization. In particular, a channel bonding and a modified medium access control procedure that distributes radio resources equitably on different data streams is proposed in Reference [22]. Another approach is to dynamically adjust the MAC layer parameters. Such as a dynamic Contention Window (CW) tuning scheme by considering the collision probability $[18,23]$, or an adaptive Arbitration Inter-Frame Space Number (AIFSN) scheme [24]. In Reference [25] the authors introduce two additional MAC-layer parameters - threshold and counter decrement value, and propose an QoS provisioning with adaptive back off algorithm in the case of multi-packet reception. Some proposed improvement methods were developed with consideration of wireless network type or its topology. Namely a dynamic assignation of both AIFSN and guidance CW depending on the number of associated stations and ACs activeness status for dense IoT-networks [19], IEEE 802.15.4 Wireless Sensor Networks [10] and for 5G-networks [20], or a signal-based MAC protocol [26] for ad hoc networks.

To enhance the quality of voice and video transmissions over IEEE 802.11 WLANs, a dynamic prediction scheme is proposed in the paper by Reference [27]. Dynamic adapting of MAC parameters (AIFSN and CW size) optimizes the access to the medium and is composed of several predictive submodels. The proposed scheme combines beacon frames, suitable separation of the AIFSN values and the appropriate selection of the CW size for each AC. For some simulated scenarios the improvement of multimedia performance is more than $20 \%$ due to reduction of the retransmission attempts.

In Reference [28], an approach and an algorithm for on-the-fly End-to-End Quality-ofService slice orchestration and IEEE 802.11 MAC management based on Software-Defined Networking principles are proposed. That allows reduce the average latency by half. Moreover, an average throughput is reduced, but less than $8 \%$.

All the mentioned works are focused on improvement of the MAC sublayer for providing QoS. To provide priority access for different types of traffic some network resources must be used, different for different implementations. Therefore, by default, the total network throughput is anyway lower than for a network with DCF scheme that does not support priority access and provides only one access category. An analysis of the impact on network throughput of replacing the non-QoS-supported DCF scheme with the QoS-supported EDCA scheme is presented in Reference [27]. The analysis is performed using simulation modeling and shows that providing priority access using EDCA for networks with five or more stations leads to decreasing of total network throughput. The paper presents an analysis of the 802.11 EDCA scheme's effectiveness, depending on the number of access categories (number of traffic priorities). The impact of varying amounts of access categories has been studied for unsaturated and saturated states of the network load. It was shown that reduction of the number of access categories improves the total network throughput, even under highly load network conditions. Although the standard EDCA scheme provides the prioritized access, for large networks EDCA is inefficient in contrast to DCF, especially when one type of traffic dominates in the network. Using EDCA with two access categories, instead of the standard four, allows to provide a good priority access for higher priority traffic.

The research described in Reference [29] is conducted with the condition that the same number of frames in each access category is received from the high-level protocols into the station queues for transmission. In real networks, such a scenario is unlikely. Wireless networks are increasingly popular with a variety of videos and voice Internet services, which add significant disparities in the ratio of high- and low-priority traffic, so investigating of wireless network performance for different traffic ratios is an important task. Therefore, this paper is focused on performance analysis of EDCA scheme depending on the different ratio of different traffic types. The throughput of wireless networks as a function of the ratio of high- and low-priority traffic for different numbers of access categories could be used for WLAN optimization based on predicted traffic types, including 
for example, the adaptive adjusting algorithm of the ACs number depending on the collected key parameters of the frames transmissions [15].

\section{Research Methodology}

To perform the study of the impact of different ratios of various traffic types on the network performance, we used a developed wireless simulator. Its accuracy was verified by comparing simulated results with the result of different analytical models [15]. The developed computer model supports the infrastructure mode, dynamic change of station load, and access models according to the 802.11 standard. A network traffic model was also developed that allows us to regulate the output loads of each station. Furthermore, the applied object-oriented programming approach provides the ability to extend or modify the standard functionalities, such as supporting of service quality, number of access categories, MAC- and physical-layer parameters, output load distribution, network load type and intensity, and network conditions.

A simulated wireless network consists of wireless stations (up to 30) and an access point, which are located within the Basic Service Set (BSS), i.e., every station is able to detect a transmission from any other station. An Infrastructure mode is considered as an operating mode for the simulated wireless network, in which all the stations communicate through a single access point. To exclude the influence of the physical environment on the network performance it is assumed, that the channel is idle. For simplicity the transmission rate is $54 \mathrm{Mbit} / \mathrm{s}$ and the other MAC and physical layers' parameters are shown in Table 1.

Table 1. Medium Access Control (MAC) and physical layers' parameter.

\begin{tabular}{cc}
\hline Frame Size & $\mathbf{2 3 1 2}$ Bytes \\
\hline MAC-header & 34 bytes \\
PHY-header & 32 bytes \\
ACK & 14 bytes \\
Slot time & $20 \mu \mathrm{s}$ \\
SIFS & $10 \mu \mathrm{s}$ \\
Retry limit & 16 \\
\hline
\end{tabular}

PHY, physical layer; ACK, acknowledgement; SIFS, Short Interframe Space.

The EDCA scheme supports four different ACs to provided QoS for different traffic types: video, voice, best-effort, and background. The first two traffic type are high-priority traffic types and require guaranteed network performance with bounded delay and jitter. These requirements are difficult to meet with network growing and increasing of network load. To study the wireless network bandwidth changing on different high- and lowpriority traffic ratios the EDCA scheme for simulated network is adjusted to support other number of ACs than only four.

\section{Simulation Scenarios and Results}

\subsection{Impact of Traffic Types Ratio on Performance of MAC Sublayer Supporting Four} Access Categories

The following four categories of network traffic are supported and processed on the MAC sublayer of a wireless LAN:

- $\quad$ Voice access category (AC_VO);

- Video access category (AC_VI);

- Best-effort (AC_BE);

- Background (AC_BK).

According to IEEE 802.11e, where the EDCA scheme with four access categories is defined, each station has four transmission queues for different types of traffic. Each AC has its own parameters, namely AIFS, CWmin, and CWmax. The DCF scheme is executed independently for each access category. Therefore, the frames from different queues may collide, and an internal collision has to be resolved by providing access to the environment 
to a frame with a higher priority, all the other internally collided frames treat internal collision as a usual collision with frames from other stations while transferring. From one side, it provides a better access for high-priority frames; from the other side, the total network performance is decreased.

To analyze wireless network performance for a different ratio of traffic types, simulations were done for two network scenarios, Scenarios A and B.

Scenario A assumed that the wireless network was mostly occupied with voice and video traffic. The ratio of traffic types is as follows:

$$
\text { AC_VO:AC_VI:AC_BE:AC_BK }=3: 3: 1: 1 \text {. }
$$

In the case of Scenario B, the ratio of traffic types was as follows:

$$
\text { AC_VO:AC_VI:AC_BE:AC_BK = 1:1:3:3, }
$$

so the network is mainly busy transmitting background or best-efforts traffic.

Normalized throughput was used as the result characteristic.

The analysis was performed for three types of networks: small, medium, and large. It was supposed that network types correspond to the number of stations, respectively, 2, 10, and 30 .

The normalized throughput for the traffic dividing scheme into four access categories for Scenarios $\mathrm{A}$ and $\mathrm{B}$ is shown in Figure 1. The Figure 1 uses the following notation: $\mathrm{AC} 3=\mathrm{AC} \_\mathrm{VO}, \mathrm{AC} 2=\mathrm{AC} \_\mathrm{VI}, \mathrm{AC} 1=\mathrm{AC} \_\mathrm{BE}$, and $\mathrm{AC} 0=\mathrm{AC} \_\mathrm{BK}$.

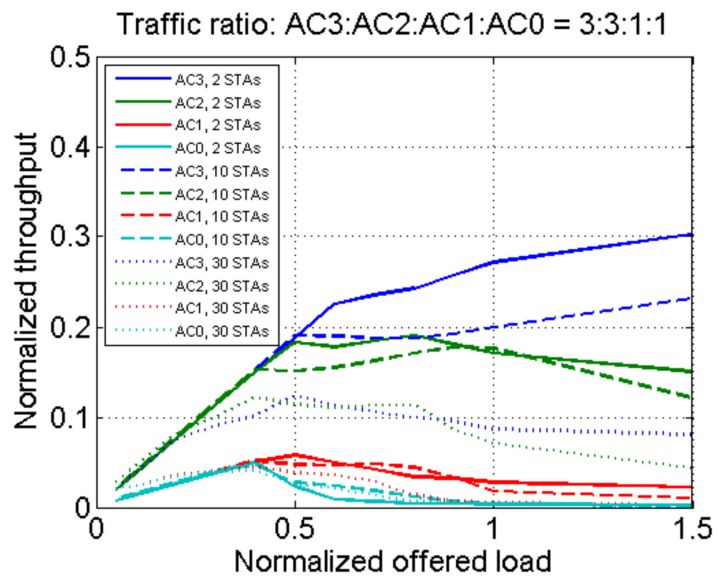

(a)

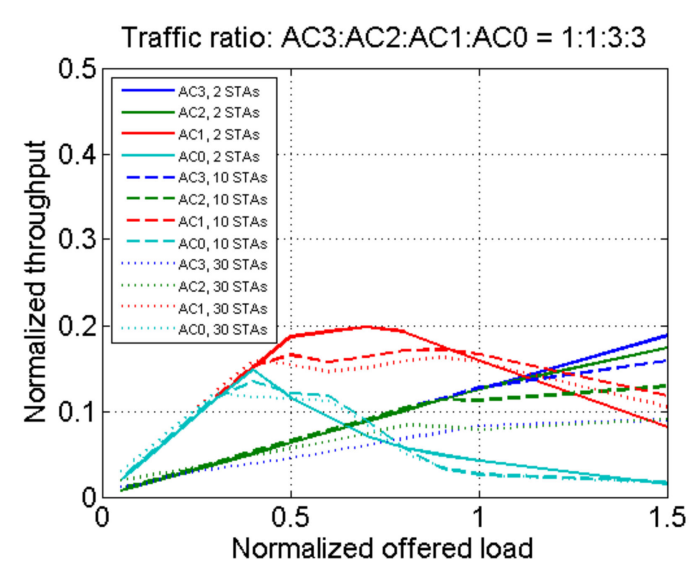

(b)

Figure 1. Normalized throughput for traffic dividing scheme onto four access categories for a network of 2, 10, and 30 stations and the traffic ratio according to (a) Scenario A and (b) Scenario B.

For Scenario A (Figure 1a), high-priority traffic is processed with the urgent access to the environment. Increasing of the number of stations in the network significantly reduces the throughput for high-priority traffic. Thus, when $80 \%$ of the network is congested, its throughput for high-priority traffic is about 1.2 and 2.4 times lower, respectively, for the network of 10 and 30 stations, as compared to a network of two stations. Note that the greater the number of stations in the network, the lower the difference between the throughput for the voice traffic AC3 and for the video traffic AC2. This most likely could be explained by increased collision probability and simultaneous access to the physical media by several stations.

For Scenario B (Figure 1b), a low-priority traffic dominates in the network. In the case of a network load below 40\%, all offered traffic (low- and high-priority traffic) is transmitted completely in the network. For the network load above $40 \%$, the amount of transmitted low-priority traffic is reduced, as compared to the amount of offered traffic. 
In this case, the EDCA scheme provides better access for the frames of the AC1 access category since their priority is higher than for the AC0 frames. For Scenario B, high-priority traffic is low (only 1/4), so the EDCA scheme almost completely allows it to be transmitted for small- and medium-sized networks. For large networks under saturation network condition, the percentage of transmitted incoming high-priority traffic is about 70\%, while, for low-priority traffic AC1, only $43 \%$ of incoming traffic is transmitted, and for the lowest priority traffic $\mathrm{AC} 0$, it is only $7 \%$.

It also can be noted that for both scenarios the amount of transmitted low-priority traffic does not depend on the network size. As it can be seen in Figure 1a, for the network of 30 stations dominated by high-priority traffic, the normalized throughput for AC3 and AC2 frames does not exceed 0.13. In the case of low-priority traffic in this network, the maximum normalized throughput for frames of access categories AC1 and AC0 (Figure 1b) is 0.16 and 0.12 , respectively.

Results of total throughput for different ratios of high- and low-priority traffic and the different number of stations are shown in Figure 2.

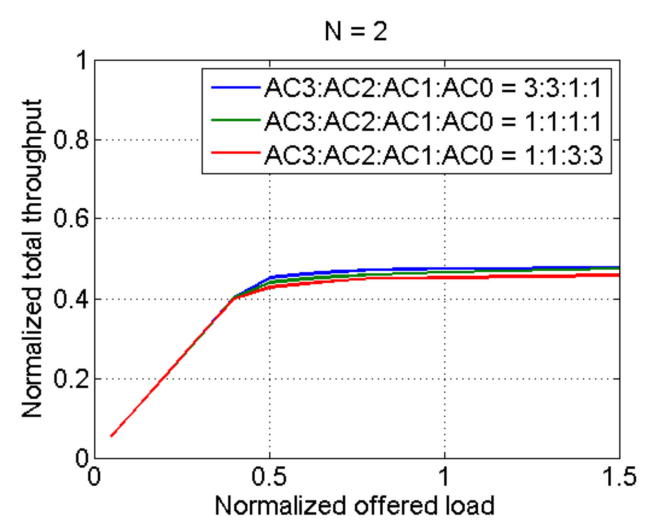

(a)

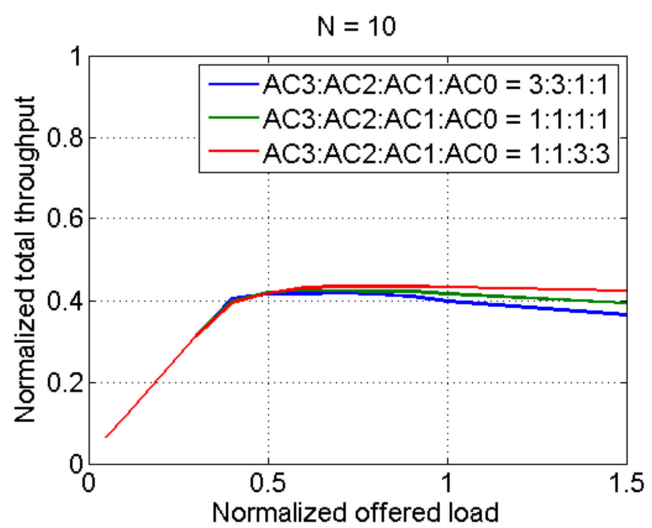

(b)

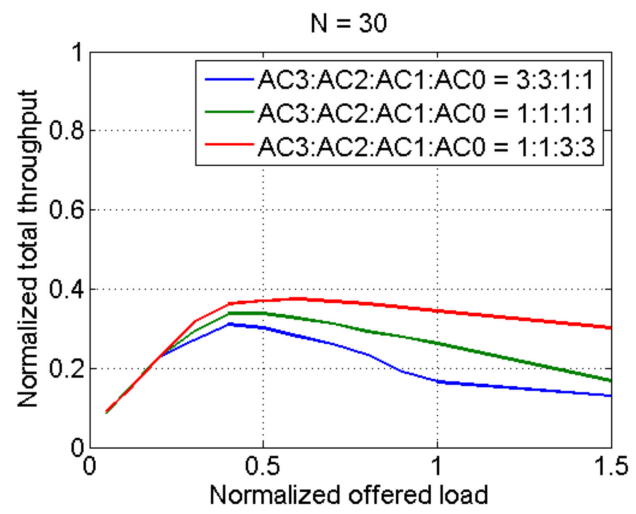

(c)

Figure 2. Total normalized throughput for traffic dividing scheme onto 4 access categories at the different traffic ratio for the network of (a) 2, (b) 10, and (c) 30 stations.

For a network that contains an access point and 2 (Figure 2a) or 10 (Figure 2b) stations, a difference in the high-/low-priority traffic ratio almost does not affect the total network throughput.

In the case of 30 stations (Figure 2c), the highest total throughput is achieved when lowpriority traffic prevails high-priority traffic in the network and vice versa the lowest total throughput is observed in the case of prevailed high-priority traffic. The more the network load is saturated, the greater is the difference between the values of total throughput, in the worst case up to almost two times. Such a difference can be explained by the fact that high-priority frames suffer from collisions. Configuration of the MAC parameter allows 
the station to get faster access to the physical environment to transmit high-priority frames and spend for such frames less time before retransmission in the case of collision. A higher number of stations in a wireless network causes a higher probability of collision itself. The maximum Contention Window (CWmax) for high-priority frames is twice smaller than for low-priority frames, and as a result, a probability that for two collided high-priority frames the same delay will be generated is much higher than for collided low-priority frames. This, in turn, leads to an increased probability that the next retransmission attempt will also be ended with a collision and that the frame will be dropped when the maximum number of retransmissions will be reached.

\subsection{Impact of Traffic Types Ratio on Quality of MAC Sublayer When Traffic Is Divided into Two Categories}

Based on the previous conclusion, that higher collision rate reduces successfully transmitted high-priority traffic, it was considered to analyze network performance, if the number of access categories is reduced to two:

- High-priority traffic, named as AC1;

- Low-priority traffic, named as AC0.

The experiments were similarly performed for the same two scenarios (A and B) when high-priority $(\mathrm{AC} 1 \mathrm{AC0}=3: 1)$ or low-priority $(\mathrm{AC1}: \mathrm{AC} 0=1: 3)$ traffic prevails in the network.

In Figure $3 \mathrm{a}$ the results for Scenario A are presented. The EDCA access scheme provides good prioritized access for high-priority frames over low-priority frames. That clearly can be observed at network load $>0.4$. The maximum throughput for small(two stations) and medium-sized (10 stations) wireless networks is about 0.45 . For large networks (30 stations) the throughput of high-priority traffic does not exceed 0.35 due to the higher probability of network collision as result of higher probability of simultaneous transmission by two or more stations in the network.

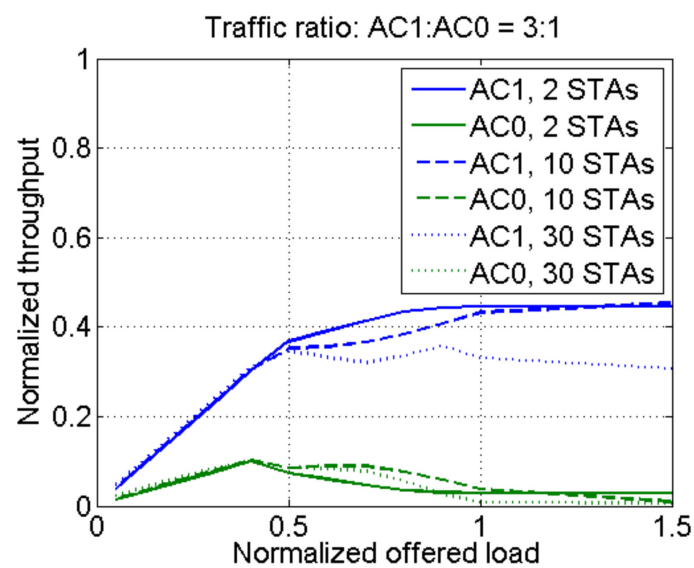

(a)

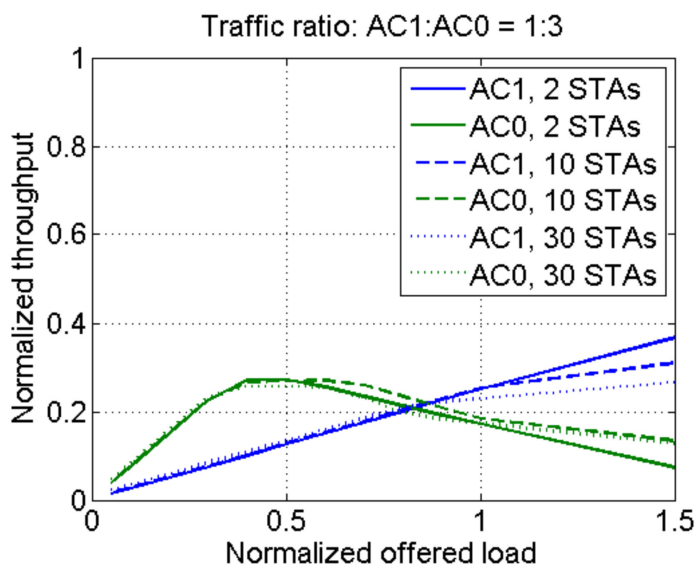

(b)

Figure 3. Normalized throughput for traffic dividing scheme onto two access categories for a network of 2, 10, and 30 stations and the traffic ratio according to (a) Scenario A and (b) Scenario B.

Prioritized access for high-priority frames is also provided in the case of Scenario B (Figure 3b) when low-priority traffic is prevalent in the network. For small networks, almost all generated high-priority frames are successfully transmitted, regardless of network load. For medium- and large-sized networks high-priority traffic ceases to be fully transmitted when network load is greater than 1 and 0.8 , respectively, due to increased probability of simultaneous environment access by two or more stations. The amount of low-priority traffic transmitted for network load $>0.4$ decreases almost linearly for network sizes.

Figure 4 shows the total normalized throughput for each type of network comparing for different traffic ratios. 


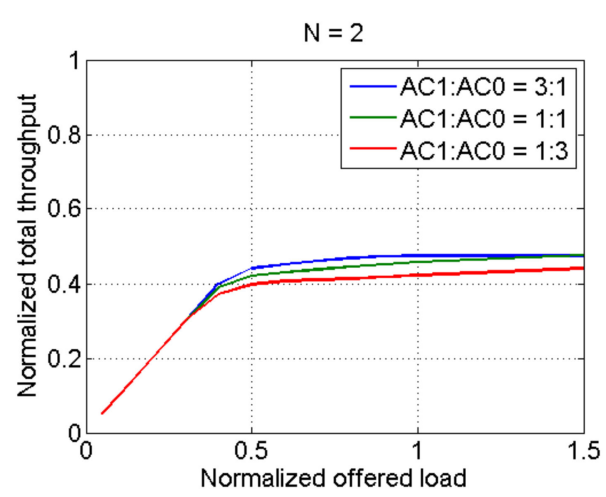

(a)

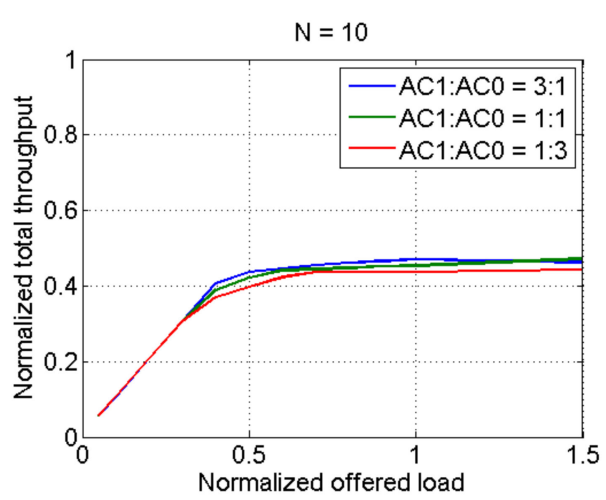

(b)

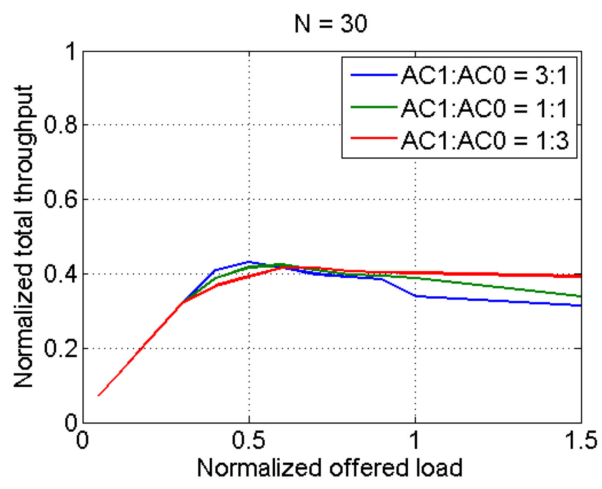

(c)

Figure 4. Total normalized throughput for traffic dividing scheme into two access categories at the different traffic ratio for the network of (a) 2, (b) 10, and (c) 30 stations.

For a medium-sized wireless network (Figure 4b) high-/low-priority traffic ratio has an insignificant effect on the total network throughput. For a wireless network that has two stations (Figure 4a), the total throughput in the case of low-priority traffic prevalence is lower on average $7-11 \%$ than for other traffic ratio scenarios.

If the number of stations in the highly loaded network is increased to 30 (Figure 4c), the total throughput of the network with prevailed high-priority traffic is less for about $12-17 \%$ than for other traffic ratio scenarios.

Providing only two access categories on the MAC sublayer for large wireless networks, if the traffic ratio of high- and low-priority traffic is significantly different, creates additional complexity to provide the quality of service for high-priority traffic such as voice and video. Increasing the high-priority traffic part in the network load might reduce the total network throughput up to $17 \%$. However, for the small- and medium-sized wireless networks, a wide scatter of high- and low-priority traffic ratio changes the network throughput is less significantly.

\section{Discussion}

\subsection{Comparison of Media Access Schemes with Two and Four Access Categories}

Summarizing the results, it can be observed that the high-/low-priority traffic ratio changes do not have a significant effect on the throughput for small and medium-sized wireless networks. However, for large wireless networks, increasing of the high-priority traffic part seriously reduces the total network throughput. The network throughput is down by almost a third (30-35\%), mainly due to high-priority multimedia traffic that, on the contrary, requires high throughput. Therefore, there is an acute need to improve the MAC-sublayer performance for wireless networks, if the high-priority traffic prevails.

Comparison of the results for access schemes with two (Figure 2c) and four (Figure 4c) access categories shows that the access scheme with four access categories provides signifi- 
cantly smaller total throughput for large wireless networks than for the access scheme with two access categories (Figure 5).

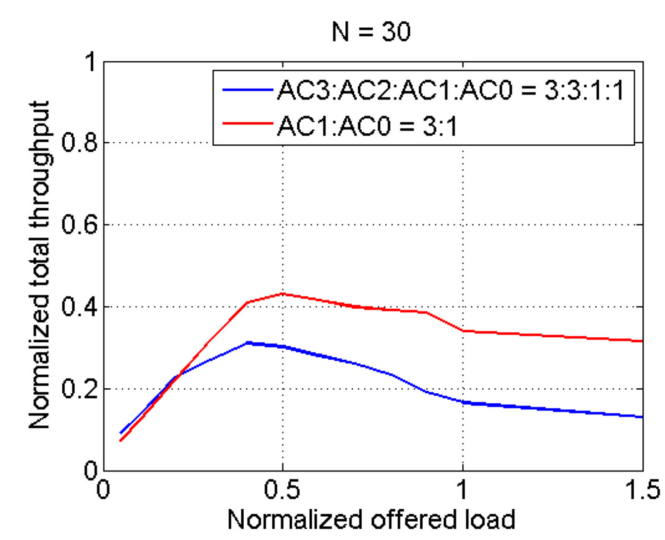

Figure 5. Total normalized throughput for traffic dividing scheme onto two and four access categories for the network of 30 stations and prevailed high-priority traffic.

For quantitative analysis of provided throughput the coefficient $\mathrm{K}_{\mathrm{RT}}$ is introduced, that determines the ratio of normalized throughput respectively for access schemes with two and four categories of access:

$$
\mathrm{K}_{\mathrm{RT}}=\mathrm{C}_{2 \mathrm{AC}} / \mathrm{C}_{4 \mathrm{AC}}
$$

where $\mathrm{C}_{2 \mathrm{AC}}$ and $\mathrm{C}_{4 \mathrm{AC}}$ are the normalized throughput values.

An analysis of the $\mathrm{K}_{\mathrm{RT}}$ coefficient values given in Table 2 shows that the total network throughput in the case of access scheme with two access categories might be even twice as high as for a standard access scheme with four access categories.

Table 2. Coefficient $\mathrm{K}_{\mathrm{RT}}$, normalized total network throughput for two and four access categories depending on the normalized offer load.

\begin{tabular}{cccc}
\hline Normalized Offered Load & $\mathbf{C}_{\mathbf{2 A C}}$ & $\mathbf{C}_{\mathbf{4 A C}}$ & $\mathbf{K}_{\mathbf{R T}}$ \\
\hline 0.05 & 0.07 & 0.09 & 0.78 \\
0.1 & 0.12 & 0.13 & 0.92 \\
0.2 & 0.22 & 0.23 & 0.96 \\
0.3 & 0.32 & 0.27 & 1.19 \\
0.4 & 0.41 & 0.31 & 1.32 \\
0.5 & 0.43 & 0.30 & 1.43 \\
0.6 & 0.42 & 0.28 & 1.50 \\
0.7 & 0.40 & 0.26 & 1.54 \\
0.8 & 0.39 & 0.23 & 1.70 \\
0.9 & 0.38 & 0.19 & 2.00 \\
1 & 0.34 & 0.17 & 2.00 \\
1.5 & 0.31 & 0.13 & 2.38 \\
\hline
\end{tabular}

5.2. Recommendations for Improving the Performance of Wireless Local Area Networks MAC Sublayer

The results of the experiments described above show that the total network throughput is highly dependent on the number of access categories used on the MAC sublayer. As the number of access categories increases, total network throughput decreases. This impact is more significant for large networks with an intensive network load, which consists mainly of the high-priority traffic. Therefore, a significant increase in the efficiency of wireless LAN under such network conditions can be achieved by improving the access scheme to the physical environment, namely using the developed algorithm of adaptive adjustment 
the number of access categories [15]. As shown in Table 1, the switching from four to two access categories can increase the total network throughput twice as high.

\section{Conclusions}

The impact of high-/low-priority traffic ratio on the MAC sublayer performance of wireless LANs is different for different network sizes and depends on the network conditions. For a standard EDCA scheme using 4 access categories, the high-/low-priority traffic ratio changes do not have a significant effect on the throughput for small and medium-sized wireless networks. However, for the large networks, this impact is significant, for example, in the case of mainly high-priority network load the total network throughput for intensive network load is almost twice as less than for the case of mainly low-priority network load.

It was also shown that the network performance using the access scheme with two access categories and with the prevailed high-priority traffic is significantly higher than for the access scheme with four categories. It allows us to conclude that improvement of the network performance, especially for large networks with the prevailed high-priority traffic, can be achieved by an adaptive adjustment of the number of access categories on the MAC sublayer.

Author Contributions: Conceptualization, K.O.; Formal analysis, O.P.; Funding acquisition, V.K.J.; Investigation, O.P.; Methodology, K.O.; Resources, V.K.J.; Software, O.P.; Supervision, K.O.; Validation, V.K.J.; Writing—original draft, K.O.; Writing—review \& editing, O.P. All authors have read and agreed to the published version of the manuscript.

Funding: This research received no external funding.

Conflicts of Interest: The authors declare no conflict of interest.

\section{References}

1. IEEE Standard for Information Technology-Telecommunications and Information Exchange between Systems-Local and Metropolitan Area Networks-Specific Requirements_Part II: Wireless LAN Medium Access Control (MAC) and Physical Layer (PHY) Specifications; IEEE Standard 802.11-2012 (Revision of IEEE Standard 802.11-2007); IEEE: New York, NY, USA, 2012.

2. Dolińska, I. The EDCA implementation in NS-3 network simulator. Zesz. Nauk. Uczel. Vistula 2018, 59, 19-29.

3. Feng, W. Performance analysis of IEEE802.11e EDCA wireless networks under finite load. Wirel. Netw. 2020, 26, 4431-4457. [CrossRef]

4. Coronado, E.; Valero, V.; Orozco-Barbosa, L.; Cambronero, M.-E.; Pelayo, F.L. Modeling and simulation of the IEEE 802.11e wireless protocol with hidden nodes using Colored Petri Nets. Softw. Syst. Model. 2020, 1-34. [CrossRef]

5. Coronado, E.; Villalon, J.; Garrido, A. Improvements to Multimedia Content Delivery over IEEE 802.11 Networks. In Proceedings of the NOMS 2020-2020 IEEE/IFIP Network Operations and Management Symposium, Budapest, Hungary, 20-24 April 2020; pp. 1-6.

6. Wang, W.-L.; Wang, C.-C.; Yao, X.-W. Slot Self-Allocation Based MAC Protocol for Energy Harvesting Nano-Networks. Sensors 2019, 19, 4646. [CrossRef] [PubMed]

7. Samara, K.; Hosseini, H. Instilling QoS in Wireless Sensor Networks. Trans. Netw. Commun. 2017, 5, 11. [CrossRef]

8. Hassan, W.H.W.; King, H.; Ahmed, S.; Faulkner, M. Enhancement techniques of IEEE 802.11 wireless local area network distributed coordination function: A review. ARPN J. Eng. Appl. Sci. 2018, 13, 1053-1062.

9. Samara, K.; Hosseini, H.; Altahat, Z.; Stewart, J.; Ehley, D.; Estrada, M. Quality of Service Provision Within IEEE 802.11 CSMA/CA Protocol. In Advances in Human Factors, Business Management, Training and Education; Metzler, J.B., Ed.; Springer: Cham, Switzerland, 2020; Volume 1129, pp. 313-319.

10. Xie, Z.; Xu, Y. Research on OTA Optimization of Wireless Sensor Networks Based on CSMA/CA Improved Algorithm. In Proceedings of the 2018 10th International Conference on Communications, Circuits and Systems (ICCCAS), Chengdu, China, 22-24 December 2018; pp. 331-335.

11. Khiat, A.; El Khaili, M.; Bakkoury, J.; Bahnasse, A. Study and evaluation of voice over IP signaling protocols performances on MIPv6 protocol in mobile 802.11 network: SIP and H.323. In Proceedings of the 2017 International Symposium on Networks, Computers and Communications (ISNCC), Marrakech, Morocco, 16-18 May 2017; pp. 1-8. [CrossRef]

12. Pérez, S.; Facchini, H.; Dantiacq, A.; Cangemi, G.; Campos, J. An evaluation of QoS for intensive video traffic over 802.11e WLANs. In Proceedings of the 2015 International Conference on Electronics, Communications and Computers (CONIELECOMP), Puebla, Mexico, 25-27 February 2015; pp. 8-15.

13. Maity, M.; Raman, B.; Vutukuru, M. TCP Download Performance in Dense WiFi Scenarios: Analysis and Solution. IEEE Trans. Mob. Comput. 2016, 16, 213-227. [CrossRef] 
14. Kryvinska, N. An analytical approach for the modeling of real-time services over IP network. Math. Comput. Simul. 2008, 79, 980-990. [CrossRef]

15. Panova, O. An Adaptive ACs Number Adjusting Algorithm for IEEE 802.11 EDCA. In Proceedings of the 8th IEEE International Conference on Intelligent Data Acquisition and Advanced Computing Systems: Technology and Applications (IDAACS'2015), Warsaw, Poland, 24-26 September 2015; pp. 823-826.

16. Thuneibat, S. A General Guide for Communication System Engineers to Improve QoS. Communications 2019, 7, 1. [CrossRef]

17. Farhana, N.; Murthy, D.H. QOS in Wireless Network-Current Trends and Future Directions. IOSR J. Comput. Eng. (IOSR-JCE) 2016, 18, 1-7.

18. Beshley, M.; Kryvinska, N.; Seliuchenko, M.; Beshley, H.; Shakshuki, E.M.; Yasar, A.-U.-H. End-to-End QoS “Smart Queue” Management Algorithms and Traffic Prioritization Mechanisms for Narrow-Band Internet of Things Services in 4G/5G Networks. Sensors 2020, 20, 2324. [CrossRef] [PubMed]

19. Salem, M.A.; Tarrad, I.F.; Youssef, M.I.; El-Kader, A.; Sherine, M. QOS categories activeness-aware adaptive edca algorithm for dense iot networks QoS Categories Activeness-Aware Adaptive EDCA Algorithm for Dense IoT Networks. Int. J. Comput. Netw. Commun. (IJCNC) 2019, 11, 67-73. [CrossRef]

20. Salem, M.A.; Tarrad, I.F.; Youssef, M.I.; El-Kader, S.M.A. An Adaptive EDCA Selfishness-Aware Scheme for Dense WLANs in 5G Networks. IEEE Access 2020, 8, 47034-47046. [CrossRef]

21. Xiao, Y.; Rosdahl, J. Performance analysis and enhancement for the current and future IEEE 802.11 MAC protocols. ACM SIGMOBILE Mob. Comput. Commun. Rev. 2003, 7, 6-19. [CrossRef]

22. Halfaoui, F.; Yazid, M.; Bouallouche-Medjkoune, L. Efficient Management of Channel Bonding in the Current IEEE 802.11ac Standard. In The Importance of New Technologies and Entrepreneurship in Business Development: In The Context of Economic Diversity in Developing Countries; Metzler, J.B., Ed.; Springer: Cham, Switzerland, 2019; Volume 102, pp. 313-321.

23. Xie, H.; Boukerche, A.; Pazzi, R.W. A novel collision probability based adaptive contention windows adjustment for QoS fairness on ad hoc wireless networks. In Proceedings of the 2012 IEEE Global Communications Conference (GLOBECOM), Anaheim, CA, USA, 3-7 December 2012; pp. 5488-5493.

24. Coronado, E.; Villalon, J.; Garrido, A. Dynamic AIFSN tuning for improving the QoS over IEEE 802.11 WLANs. In Proceedings of the 2015 International Wireless Communications and Mobile Computing Conference (IWCMC), Dubrovnik, Croatia, 24-28 August 2015; pp. 73-78.

25. Arun, I.B.; Venkatesh, T.G.; Dappuri, B. QoS provisioning with adaptive backoff algorithm for IEEE 802.11ac under multipacket reception. In Proceedings of the 2014 9th International Symposium on Communication Systems, Networks \& Digital Sign (CSNDSP), Piscataway, NJ, USA, 23-25 July 2014; pp. 826-830.

26. Kosek-Szott, K.; Natkaniec, M.; Pach, A.R. A new busy signal-based MAC protocol supporting QoS for ad-hoc networks with hidden nodes. Wirel. Netw. 2013, 19, 1135-1153. [CrossRef]

27. Coronado, E.; Villalon, J.; Del Solo, A.G. An Adaptive Medium Access Parameter Prediction Scheme for IEEE 802.11 Real-Time Applications. Wirel. Commun. Mob. Comput. 2017, 2017, 1-19. [CrossRef]

28. Isolani, P.H.; Cardona, N.; Donato, C.; Marquez-Barja, J.; Granville, L.Z.; Latre, S. SDN-based Slice Orchestration and MAC Management for QoS delivery in IEEE 802.11 Networks. In Proceedings of the 2019 Sixth International Conference on Software Defined Systems (SDS), Rome, Italy, 10-13 June 2019; pp. 260-265.

29. Leontyeva, O.; Obelovska, K. Performance analysis of IEEE 802.11 EDCA for a different number of access categories and comparison with DCF. In Proceedings of the 20th International Science Conference: Computer Networks CN 2013, Lwówek Śląski, Poland, 17-21 June 2013; pp. 95-104. 